\title{
El equipo de investigación: un reto y una realidad
}

\author{
Magdalena Alfaro Rodríguez. \\ Centro de Investigación y Docencia en Educación (CIDE), Universidad Nacional \\ Heredia, Costa Rica
}

Alejandra Gamboa Jiménez²

Centro de Investigación y Docencia en Educación (CIDE), Universidad Nacional

Heredia, Costa Rica

Susana Jiménez Sánchez $z^{3}$

Centro de Investigación y Docencia en Educación (CIDE), Universidad Nacional

Heredia, Costa Rica

Jorge R. Martín Pérez ${ }^{4}$

Centro de Investigación y Docencia en Educación (CIDE), Universidad Nacional

Heredia, Costa Rica

Andrea Ramírez González ${ }^{5}$

Centro de Investigación y Docencia en Educación (CIDE), Universidad Nacional

Heredia, Costa Rica

\begin{abstract}
${ }^{1}$ Magíster en Educación con énfasis en Docencia Universitaria de la Universidad Nacional, Costa Rica. Licenciada en Música con énfasis en Educación Musical. Actualmente, es académica de la División de Educología del Centro de Investigación y Docencia en Educación (CIDE) de la Universidad Nacional, Costa Rica, y es integrante del cuarto equipo del Proyecto Perfiles, dinámicas y desafíos de la educación costarricense. Correo electrónico: magdar613@hotmail.com
\end{abstract}

${ }^{2}$ Máster en Psicopedagogía de la Universidad de La Salle, con estudios en Ciencias de la Educación con énfasis en Orientación de la Universidad Nacional, Costa Rica, actualmente es la subdirectora de la División de Educación para el Trabajo del Centro de Investigación y Docencia en Educación (CIDE) de la Universidad Nacional, Costa Rica y fue integrante del tercer equipo del proyecto Perfiles, dinámicas y desafíos de la educación costarricense. Correo electrónico: 1gamboa@una.ac.cr

\footnotetext{
${ }^{3}$ Máster en Tecnología Educativa de la Universidad Estatal a Distancia, Costa Rica. Licenciada en Enseñanza de la Química de la Universidad de Costa Rica y en Ciencias de la Educación con énfasis en Docencia de la Universidad Estatal a Distancia, Costa Rica. Con estudios en educación primaria. Actualmente, es académica de la División de Educología del Centro de Investigación y Docencia en Educación (CIDE) de la Universidad Nacional, Costa Rica y coordinó el tercer equipo del proyecto Perfiles, dinámicas y desafíos de la educación costarricense. Correo electrónico: sujisa@gmail.com
}

${ }^{4}$ Magíster en Educación con énfasis en Docencia Universitaria de la Universidad Nacional, Costa Ria, Licenciado en Economía y Licenciado en Cibernética Matemática de la Universidad de la Habana, Cuba. Actualmente, es académico de la División de Educación para el Trabajo del Centro de Investigación y Docencia en Educación (CIDE) de la Universidad Nacional, Costa Rica y coordina el cuarto equipo del proyecto Perfiles, dinámicas y desafíos de la educación costarricense. Correo electrónico: jorgeramonmartin@yahoo.es

${ }^{5}$ Licenciada en Enseñanza de la Matemática de la Universidad de Costa Rica y con estudios en Educación Primaria. Actualmente, es académica de la División de Educación Rural del Centro de Investigación y Docencia en Educación (CIDE) de la Universidad Nacional, Costa Rica y fue integrante del tercer equipo del proyecto Perfiles, dinámicas y desafíos de la educación costarricense.

Correo electrónico: aramgo@costarricense.cr

La Revista Electrónic@ Educare está indizada en el Catálogo LATINDEX.

Artículo protegido por la licencia Creative Commons. 


\author{
Marie-Claire Vargas Dengo 6 \\ Centro de Investigación y Docencia en Educación (CIDE), Universidad Nacional \\ Heredia, Costa Rica \\ Elionay Quirós Meneses ${ }^{7}$ \\ Centro de Investigación y Docencia en Educación (CIDE), Universidad Nacional \\ Heredia, Costa Rica
}

Recibido 10 de setiembre de 2009 • Aceptado 02 de diciembre de 2009

\begin{abstract}
Resumen. En este ensayo se relatan los retos iniciales, los procesos de trabajo, la conformación como equipo y los aprendizajes obtenidos por investigadores e investigadoras del equipo interdisciplinario del Proyecto Perfiles, Dinámicas y Desafíos de la Educación Costarricense del Centro de Investigación y Docencia en Educación (CIDE) de la Universidad Nacional, en su tercera etapa. Se analiza cómo el trabajo cooperativo y el liderazgo compartido como equipo, hicieron exitoso al proceso de investigación.
\end{abstract}

Palabras clave. Equipo, investigación, liderazgo, trabajo cooperativo.

Abstract. This paper presents the different initial challenges, work processes, team formation and learning content obtained by researchers of the interdisciplinary team of the project called Proyecto Perfiles, Dinámicas y Desafíos de la Educación Costarricense (Profiles, Dynamics and Challenges of the Costa Rican Education) of Centro de Investigación y Docencia en Educación (CIDE) (Research and Teaching Center), from Universidad Nacional, in its third phase. There will be an analysis showing how cooperative work and shared leadership as a team made a successful impact in the research process.

Key words. Team, research, leadership, cooperative work.

\title{
Introducción
}

¿Qué hace la diferencia entre los equipos que entran en órbita y logran una sinergia real y los que fracasan y desaparecen? La respuesta se relaciona mucho más con cómo los equipos están estructurados y apoyados que con sus virtudes propias o con las obligaciones que puedan tener como unidades de funcionamiento. (Hackman, citado por Hesselbein y Cohen, 2002, p. 391)

Cuando decidimos realizar una investigación de cualquier tipo, uno de los elementos que más nos hacen pensar sobre su futuro, es el equipo con el que trabajaremos. ¿Qué pasa si nos eligen para participar en una investigación con un grupo del cual no conocemos a las personas con las que nos corresponderá trabajar?

Los investigadores y las investigadoras del equipo interdisciplinario del proyecto Perfiles, Dinámicas y Desafíos de la Educación Costarricense (PDDEC), del Centro de Investigación y

\footnotetext{
${ }^{6}$ Máster en Currículum e Instrucción de The George Washington University, USA. Actualmente, es académica de la División de Educación Básica del Centro de Investigación y Docencia en Educación (CIDE) de la Universidad Nacional, Costa Rica y es integrante del cuarto equipo del Proyecto perfiles, dinámicas y desafíos de la educación costarricense. Correo electrónico: $\underline{\text { educare@una.ac.cr }}$

${ }^{7}$ Máster en Tecnología e Informática Educativa de la Universidad Nacional de Costa Rica. Actualmente, es académico de la División de Educología del Centro de Investigación y Docencia en Educación (CIDE) de la Universidad Nacional, Costa Rica y fue integrante del tercer equipo del proyecto Perfiles, dinámicas y desafíos de la educación costarricense. Correo electrónico: equiroster@gmail.com
} 
Docencia en Educación (CIDE) de la Universidad Nacional, viven esta situación. Son seleccionados en sus unidades académicas para conformar un grupo de trabajo con personas con las que nunca han compartido responsabilidades académicas.

Entonces, vale la pena preguntarse: ¿de qué forma logran organizarse y estructurarse como un equipo de trabajo que aprende y crece? Al respecto, destacamos las ideas de Senge (2006) quien afirma que en toda organización que aprende, las personas deben tener la oportunidad de crecer y de desarrollarse.

Por ello, según Senge (2006), todo equipo bien organizado tiene un aprendizaje en tres dimensiones, a saber:

1. La necesidad de pensar, agudamente, sobre los problemas complejos. Se aprende a pensar como colectividad.

2. La necesidad de una acción innovadora y coordinada. Se comprende que los actos de una persona complementan los actos de los demás.

3. El papel de los miembros del equipo en otros equipos. Un equipo que aprende alienta a otros a conformar una organización similar.

Creemos que, como investigadores e investigadoras de la tercera etapa del PDDEC, logramos obtener estos tres tipos de aprendizaje. Sin embargo, lograr esto fue todo un reto, pero también una realidad. Relatar los retos iniciales, los procesos de trabajo, la conformación como equipo y los aprendizajes que obtuvimos las personas que conformamos el equipo investigador, son los objetivos de este escrito.

\section{Antecedentes}

El PDDEC del CIDE nace en el año 2002, cuando se aprueba la conformación de un equipo de investigación, en el que todas las unidades académicas del CIDE tuvieran una persona que los representase. Se designa al Decanato de este Centro como el encargado de albergar el proyecto por realizar.

Fue en 2003 cuando inició la tarea del primer grupo de investigadores, quienes se plantearon en su primera etapa (2003-2006) realizar un análisis de la situación del sistema educativo costarricense. La investigación buscó ofrecer información y análisis en torno a la evolución histórica, estructura, dinámicas vigentes y desafíos de los diferentes niveles del sistema educativo formal: preescolar, primero, segundo y tercer ciclos de la educación general básica, educación diversificada y educación superior.

Este primer grupo de académicos y académicas, al relatar su experiencia como grupo de investigadores, señalaron los siguientes elementos que ayudan a facilitar la consolidación de un equipo: a) el conocimiento de las personas participantes, b) el planteamiento de las expectativas, c) la expresión de los temores de los participantes, d) la promoción de un pensamiento compartido, e) la búsqueda de metas e ideales comunes (Esquivel, Induni, Madrigal, Pereira, Solano y Umaña, 2004).

En la segunda etapa del proyecto (2006-2007), se corroboró la importancia de diseñar y ejecutar un estudio que permitiera comprender, con mayor precisión, cuáles son los factores que facilitan la permanencia de los y las jóvenes en el sistema educativo, particularmente, en la transición de la primaria a la secundaria. El estudio se centró en los factores que contribuyen a la permanencia y avance de los y las estudiantes en el Tercer Ciclo de la Educación General Básica, particularmente en el sétimo año. 
Con base en los resultados de la segunda etapa, el tercer equipo investigador (2007-2008) se interesó en caracterizar el perfil de los y las docentes de sétimo año, de manera que pudieran identificarse aquellos factores claves que propician una relación interpersonal positiva entre docentes y estudiantes, y que contribuyen en la transición, la permanencia, el mejoramiento de la promoción y el éxito de los y las estudiantes dentro del sistema educativo formal. Su producto fue una propuesta del perfil docente de sétimo año, acorde con las necesidades y las demandas actuales del país. Esta propuesta se puso a disposición de las instancias correspondientes del Consejo Nacional de Rectores, del Ministerio de Educación Pública y de las universidades estatales y privadas.

\section{La evolución del equipo de trabajo}

En diciembre de 2006, se reunieron por primera vez cinco de los y las integrantes del equipo de investigación de la tercera etapa del proyecto; sin embargo, fue hasta febrero de 2007 cuando se seleccionó la coordinadora y fue en este mes, también, cuando se reunió el equipo completo.

El trabajo se inició con seis personas: una de la División de Educación para el Trabajo, una de la División de Educación Rural, una de la División de Educación Básica, una del Instituto de Estudios Interdisciplinarios de la Niñez y la Adolescencia y dos de la División de Educología, ya que, a ésta última unidad le correspondía la coordinación de esta etapa; al respecto, cabe rescatar que en cada etapa de investigación le corresponde a una unidad académica la coordinación del equipo. Cinco personas se mantienen durante todo el proceso de investigación, en febrero de 2008, una de las compañeras del equipo original, deja el proyecto, por lo que se divide ese tiempo y se incorporan dos nuevos miembros. Asimismo, en agosto de 2007, se incorpora una nueva participante (por cuatro meses), cuyo objetivo era brindar apoyo al equipo, en un área específica.

De esta forma, el equipo tuvo un total de ocho investigadores e investigadoras quienes afianzaron sus conocimientos en la formulación, la gestión, el desarrollo y la evaluación del proceso investigativo.

\section{Construyendo el proyecto de investigación}

Desde la primera reunión, el equipo de trabajo decide analizar los informes de investigación de las dos etapas anteriores, de manera que se tuviera un panorama general de la historia del proyecto y, de esta forma, se plantea la posibilidad de proponer una investigación que diera continuidad a los tópicos de las investigaciones anteriores, y se toma la primera decisión consensuada, la cual consistió en:

1. Elaborar una primera propuesta de investigación, con respecto a la formación de los y las docentes de sétimo año.

2. Investigar sobre el perfil del docente para sétimo año.

Esta segunda propuesta de investigación fue avalada por el Consejo Académico del CIDE, fue revisada en un proceso en espiral, en el que, continuamente, se revisaron los objetivos, los referentes teóricos, la metodología y los instrumentos, a partir de la reflexión dentro del equipo y la consulta realizada a expertos nacionales e internacionales, en el ámbito de la investigación educativa. 
El equipo estuvo dispuesto a escuchar las sugerencias de estos expertos y a incorporar aquellas que consideraron pertinentes para el diseño y el proceso de investigación. Asimismo, al estar en contacto con los y las docentes, administrativos y el estudiantado de sétimo año, se realizaron ciertas adecuaciones metodológicas durante el proceso de indagación para favorecer y facilitar el análisis de los resultados encontrados.

\section{El grupo de investigadores seleccionado como equipo}

Un equipo es más que la suma individual de quienes lo conforman, ya que, al tener una visión compartida, se trabaja de forma cooperativa y emocionalmente inteligente; es un conjunto de personas diversas que se organizan con el fin de lograr un objetivo. Al formar un conjunto uniforme, lo que le ocurra a alguno de los miembros afecta a los demás y, por tanto, al trabajo que se está realizando. En un equipo se requiere de un trabajo cooperativo, en el que todos sus miembros realizan aportes para la consecución de las metas. Tal y como lo dijo uno de los miembros de este grupo de investigadores:

La experiencia de formar parte del Proyecto Perfiles, Dinámicas y Desafíos de la Educación Costarricense en su III etapa, la describo como una experiencia muy enriquecedora tanto para el crecimiento personal como profesional de cada uno de los miembros del grupo. Desde un inicio, este equipo de trabajo, reunido con un claro y definido objetivo en común, se caracterizó por ser un equipo colaborativo, más que autocrático, en el que cada miembro contribuyó con aportes relevantes y pertinentes desde sus capacidades, conocimientos y disposiciones. Como grupo, vivenciamos la creatividad como un proceso colectivo y social que generó propuestas y productos académicos específicos. (Alfaro et al, 2008, I 1)

El equipo emerge de la interacción de las personas, lo que permite a todos sus miembros evolucionar mediante una dinámica que ningún otro equipo tendrá, es decir, cada equipo es único e irrepetible. Asimismo, la gestión dentro del equipo de investigación, implicó poner en común acuerdo algunas decisiones que resultaban trascendentales a lo largo del proceso. Gracias a esta concepción, se permitió a todas las personas que conformaron el equipo, dar a conocer sus puntos de vista y cómo visualizaban el trabajo que se estaba realizando.

Sobre la base de altos valores, tales como el respeto a la diversidad, la solidaridad y el compromiso, que sustentan la plataforma del trabajo cooperativo, cada miembro del equipo se comprometió a realizar ciertas tareas, compartir las responsabilidades de otras y poner en común, constantemente, el trabajo realizado, con lo que se lograron los objetivos propuestos y aún más de los que se habían planteado al principio.

De acuerdo con Goleman, Boyatzis y McKee (2006), un equipo emocionalmente inteligente reconoce sus capacidades, sus puntos fuertes y sus debilidades, siendo cada uno de sus miembros capaz de realizar una lectura del clima emocional imperante y de responder de manera adecuada a éste. Como dicen estos autores: "las personas trabajan mejor cuanto mejor se sienten" (p. 43), tal y como se destaca en el siguiente relato ofrecido por uno de los miembros del equipo: "Realmente, la participación en este especial colectivo me ha asentado hasta el sentido de pertenencia a la institución, descubriéndome la importancia del liderazgo democrático, motivador y realmente abierto a iniciativas y creatividad" (Alfaro et al, 2008, I 3). 
Hackman (citado por Hesselbein y Cohen, 2002) afirma que un equipo exitoso debe tener:

1. Un objetivo que se preste al trabajo en equipo

2. Una dirección clara y atractiva

3. Una estructura que promueva un trabajo en equipo competente

4. Algunas recompensas, sistemas de educación e información adaptados

5. Un entrenamiento para ayudar a los miembros a aprovechar las circunstancias favorables

Por lo anteriormente expuesto, y según las evaluaciones constantes realizadas por este equipo de trabajo se establece que, además de estos aspectos exitosos, el equipo de investigación debe tener un líder empoderado en las metas de trabajo, con apertura a la diversidad de criterios académicos y con una visión compartida de los valores propios del equipo.

La interdependencia, el alto nivel de confianza y la participación de cada miembro, hacen que el equipo se estructure como una unidad inteligente:

Formar parte del equipo de investigación en el Proyecto Perfiles, Dinámicas y Desafíos de la Educación Costarricense resultó una experiencia muy interesante: en primer lugar, porque desde el tema escogido hasta las conclusiones del informe fueron elaborados bajo el consenso de todos los participantes, no hubo en ningún momento imposición de ninguno de los integrantes. El trabajo en equipo nos caracterizó en todos los momentos del proceso... Una de las cosas que nos ayudó enormemente fue que todos(as) estábamos muy comprometidos(as) con el tema y asumimos la investigación con verdadera pasión. Pasión que debe caracterizar a todo investigador. (Alfaro et al, 2008, I 4)

La diferencia existente entre los equipos de mayor rendimiento y aquellos otros cuyo rendimiento no supera el promedio radica en su inteligencia emocional. La inteligencia emocional del grupo determina su capacidad para gestionar sus emociones de un modo que aliente "la confianza, la identidad y la eficacia grupal maximizando, así, en consecuencia, la cooperación, la colaboración y la eficacia" (Goleman et al., 2002, p. 223). Dijo una de las investigadoras:

Aprendí a trabajar en equipo, a respetar las ideas ajenas, a conocer mis capacidades, a consensuar, a trabajar con eficiencia y rapidez; a sacar las tareas sin dejar que se me acumularan; pero, también, a realizar tareas sin esperar que me las pidieran; aunque, en general, estaban muy bien definidas, a adelantar trabajo, a plantear propuestas, a plantear ideas sobre cómo llevar a cabo el trabajo, a leer rápido y entresacar las ideas que nos podían servir para el trabajo. Aprendí a valorar las capacidades de mis compañeras y compañeros, de algunas lo ordenadas, de otras lo ejecutivas, de otras la capacidad de análisis y de otras la capacidad para ordenar las ideas y de tener los esquemas claros en la construcción de un libro o del informe. Por último, a respetar el pensamiento divergente y a poder consensuar. (Alfaro et al, 2008, I 2)

\section{El liderazgo del equipo en el éxito de la investigación}

El líder establece el rumbo y el objetivo con su historia, su sentido del propósito, sus valores y su visión (Senge, 2006, p. 440). Generalmente, se sitúa al liderazgo en una persona; no obstante, 
trabajar en un equipo de investigación como el que se encuentra en mención, corrobora que el liderazgo se vive y se comparte gracias a las capacidades humanas que poseen los miembros del equipo. Con base en el principio de interdependencia que se da entre los grupos humanos, en los que cada miembro es importante y necesario, Kerr (citado por Hesselbein y Cohen, 2002) señala que los líderes eficaces saben que no pueden trabajar solos, ya que, como personas, creamos relaciones de interdependencia, por lo que la complejidad está a la orden del día.

Beers (citado por Hesselbein y Cohen, 2002) dice que "definir lo que se hace, por qué existe y cómo se aprecia su éxito es tarea del líder" (p. 289), es decir, que la gente necesita sentirse identificada con su equipo de trabajo y requiere establecer lazos emocionales con quienes le rodean; y es el líder quien debe afianzar ese sentido de pertenencia en cada una de las personas que trabajan con él. Es así cómo, en la madurez del equipo de investigación, se van generando alianzas estratégicas, afectos e intereses académicos.

El liderazgo en el equipo de investigación se consolida con la suma de los líderes que lo conforman, con la claridad de la gestión que ejerce la figura de la coordinación, quien, además de llevar la visión de forma clara, también, desempeña funciones de gestión administrativa.

Los líderes efectivos que realizan investigación poseen las siguientes características:

1. Buscan consensos, en medio de la diversidad de criterios en la que se desarrolla la investigación.

2. Poseen humildad académica y personal, para poder dar sus criterios y nutrirse del de los demás.

3. Se apoyan como equipo y no como un grupo seleccionado para un fin; al consolidarse el equipo, dejan de ser un grupo de investigadores y pasan a ser un equipo que investiga con madurez.

4. Se plantean retos que aporten a la comunidad científica para la mejora de una ciencia o una disciplina específica, las metas no sólo tienen un propósito limitado hacia una organización, sino que presentan el desafío de colaborar a la academia y a la comunidad científica.

5. Sus fortalezas y debilidades son conocidas por el equipo, lo cual es producto de la madurez que da el tiempo que dura el proceso de investigación; él sabe, con claridad, quiénes pueden aportar en un área u otra.

6. Manifiestan sinergia y se sobreponen en la adversidad; utilizan las posibilidades y las circunstancias para armonizar los espacios que se tienen para trabajar en la investigación; y cuando se presentan las adversidades propias del proceso, el equipo logra sobreponerse.

7. Comparten con el equipo los logros de la investigación.

Son tan variadas las experiencias y los aprendizajes de quienes formaron parte del equipo, que queremos dejar plasmadas algunas de sus opiniones:

Me ha enriquecido mucho esta especial experiencia de investigación mixta, aunque con énfasis en el enfoque cualitativo, como integrante de un equipo multidisciplinario tanto por la formación como por las experiencias de cada participante, con destaque de la calidad humana, honestidad y ética profesional, humildad y elevado sentido de la responsabilidad de cada integrante, aunado todo a un ambiente de destacado espíritu colaborativo en el que todas y todos, con nuestros criterios sumamos siempre, aun cuando las resultantes finales no se parecieran a ninguno de los aportes específicos. (Alfaro et al, 2008, I 7)

La determinación para adaptarme al grupo de trabajo y hacer mis aportes de la mejor manera, fue, en primer lugar, encontrarme un EQUIPO DE TRABAJO bien constituido, en el que 
no solamente persistía el trabajo como único elemento, sino el espacio de trabajo y el buen ambiente que se vivía. Lo segundo es, propiamente, la actitud con la que llegué, pues era claro que mi intención era la de aportar al máximo, y la humildad para lograr adaptarme, con mayor facilidad, al equipo de trabajo. (Alfaro et al, 2008, I 6)

A pesar de tener una función bien definida, me sentí motivacionalmente bien, cuando tuve la oportunidad de participar en las giras y colaborar en otras acciones encomendadas. Estas cosas fueron las que me permitieron sentirme parte de este equipo. Sobre todo, porque se hacían con voluntad de todas y todos, la disposición de cada uno se reflejaba cuando la coordinadora delegaba funciones en los miembros participantes. (Alfaro et al, 2008, I 9)

Cuando las personas sienten que están haciendo un trabajo de valor, buscan la forma de hacerlo bien y de mejorar día con día, si conocen las metas y los objetivos del grupo y si saben que, como equipo, su trabajo y sus decisiones influyen en las demás personas, intentarán establecer relaciones de trabajo emocionalmente sanas.

\section{Reflexiones finales}

Como reflexiones finales, se aporta una serie de aspectos que contribuyeron al éxito de las metas planteadas por el equipo de investigación:

Tuvimos un sueño compartido. Contar con un proceso de creación de una visión del futuro, la cual tuvo en cuenta los intereses y las diversidades de las y los integrantes del equipo, fue fundamental. Esto promovió la motivación entre las y los participantes.

Produjimos resultados observables. Logramos construir no sólo un conjunto de productos teóricos, sino, también, fortalezas derivadas de la confianza, consideración y respeto mutuos con mucha afectividad, todo lo que, al cabo, nos permite disfrutar una mezcla de éxitos, experiencias y mayor autoestima.

Manifestamos el liderazgo del equipo. Logramos manifestar un liderazgo participativo y promotor de las habilidades de cada uno de los miembros del equipo.

Nos dimos apoyo mental, emocional y desarrollamos compañerismo. Fue preciso hallar canales de comunicación tanto en un nivel formal como informal, en un ambiente de trabajo de respeto y cordialidad.

Cooperamos el uno con el otro. Fuimos conscientes, desde el momento que empezamos a trabajar como equipo, que es fundamental llegar a consensos. Pero lo más importante, en este sentido, es que cada persona tuvo el compromiso de colaborar, de ofrecer sus habilidades para el servicio de los demás y de poner en práctica la humildad personal y académica.

Nos apoyamos en nuestras virtudes. Se resaltaron los aportes de cada uno de los miembros, de acuerdo con lo que le correspondía y con sus capacidades. De esta forma, las metas se convirtieron en éxito, pero eso se logra sólo con el apoyo de todos los miembros del equipo. 
Consideramos, también, que nos encontramos dentro de un grupo de mujeres y hombres, de diferentes edades, con habilidades y formaciones profesionales diferentes, lo que nos hizo aprender unos de otros. Por tanto, creemos que es necesario que las experiencias como las que se adquieren en el Proyecto Perfiles, Dinámicas y Desafíos de la Educación Costarricense sean incentivadas no sólo en el CIDE, sino en toda la Universidad Nacional.

\section{Referencias bibliográficas}

Alfaro, M., Gamboa, A., Jiménez, S., Martín, J., Ramírez, A., Quirós, E. et al. (2008). Discusión grupal sobre las experiencias personales en el Proyecto Perfiles, Dinámicas y Desafíos de la Educación Costarricense. Heredia, Costa Rica.

Cirigliano, G. \& Villaverde, A. (1994). Dinámica de grupos y educación. (20ª ed.) Buenos Aires, Argentina: Editorial Hvmanitas.

Esquivel, L.; Induni, G.; Madrigal, V.; Pereira, Z.; Solano, J. \& Umaña, W. (2004). Conformación de un equipo de investigadores: una experiencia concreta. Revista Educare, Vol. 6, № 1, 193204.

Goleman, D., Boyatzis, R. \& McKee, A. (2002). El líder resonante crea más. (1ª Reimpres). México, D. F.: Random House Mondadori.

Hesselbein, F. \& Cohen, P. (2002). De líder a líder. Buenos Aires, Argentina: Granica.

Senge, P. M. (2006). La quinta disciplina: el arte y la práctica de la organización abierta al aprendizaje. Buenos Aires, Argentina: Granica.

\section{Otra fuente consultada}

Yukl, G. (2006). Leadership in organizations. (6 $6^{\text {th }}$ ed.). Upper Saddle River, N. J. U. S. A.: Prentice Hall. 